POPULATIONS Populations vulnérables

VULINERABLES $\quad 4 \mid 2018$

Jeunes vulnérables. Incidences sur les parcours

d'entrée dans la vie adulte

\title{
Ségrégations et parcours professionnalisés des sortants du supérieur
}

Segregation and career paths of higher education graduates

Philippe Lemistre

\section{OpenEdition}

Journals

Édition électronique

URL : https://journals.openedition.org/popvuln/824

DOI : $10.4000 /$ popvuln. 824

ISSN : 2650-7684

Éditeur

LIR3S - Laboratoire Interdisciplinaire de Recherche "Sociétés Sensibilités Soin" (UMR 7366 CNRS-uB)

Édition imprimée

Date de publication : 1 juin 2018

Pagination : 115-135

ISBN : 978-2-918173-23-6

ISSN : 2269-0182

Référence électronique

Philippe Lemistre, « Ségrégations et parcours professionnalisés des sortants du supérieur 》,

Populations vulnérables [En ligne], 4 | 2018, mis en ligne le 12 juillet 2019, consulté le 26 février 2022.

URL : http://journals.openedition.org/popvuln/824 ; DOI : https://doi.org/10.4000/popvuln.824

Ce document a été généré automatiquement le 26 février 2022.

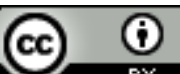

Les contenus de la revue Populations vulnérables sont mis à disposition selon les termes de la Licence Creative Commons Attribution 4.0 International 


\title{
Ségrégations et parcours professionnalisés des sortants du supérieur
}

Segregation and career paths of higher education graduates

\author{
Philippe Lemistre
}

\section{Introduction}

1 Les pouvoirs publics affichent une nouvelle ambition de «bac moins trois à bac plus trois» pour établir "un continuum de formation articulant les trois années qui précèdent et les trois années qui suivent le baccalauréat » (circulaire MEN-MESR $n^{\circ}$ 2013-2012 du 18/06/2013). Pour renforcer l'égalité des chances, il faut alors permettre à tous d'accéder à un niveau d'études de trois années après le bac au moins. Afin d'adapter l'offre à l'ensemble des publics et permettre au plus grand nombre d'accéder à l'enseignement supérieur, la création de filières professionnelles ou de diplômes à bac plus trois et plus semble essentielle (Lichtenberger et Korolitski, 2012).

2 Il s'agit de permettre des parcours diversifiés où les bifurcations sont facilitées, notamment entre filières générales et professionnelles. Les filières dites professionnelles sont clairement identifiées par les institutions, du Certificat d'aptitude professionnelle (CAP) au Master professionnel. Ces diplômes professionnels n'ont eu de cesse de se multiplier, notamment par la création des baccalauréats professionnels en 1985, puis des licences professionnelles en 1999. C'est une définition restrictive du caractère professionnel des filières qui correspond à une acception institutionnelle, même si la frontière entre général et professionnel n'est pas dans les faits aussi claire ${ }^{1}$.

3 La création de diplômes, de filières et de spécialités de formation va de pair avec une sélectivité accrue des publics. Ainsi, la multiplication des titres brouillerait les cartes sans jamais être amenée à les redistribuer, un phénomène qui participe à la reproduction sociale en «jouant des erreurs de perception que favorise la floraison 
anarchique des filières et des titres à la fois relativement insubstituables et subtilement hiérarchisés » (Bourdieu, 1979, p. 174). C'est la démocratisation ségrégative (Merle, 2012). Cette notion met en évidence ce que peut masquer la démocratisation quantitative, supposée uniforme, au sens d'une augmentation généralisée des niveaux d'éducation qui en réalité masque des logiques internes à chaque niveau. Alors qu'auparavant les enfants de milieux riches en capitaux économiques et culturels se distinguaient par des durées de scolarité plus longues (inégalités quantitatives), ils se distinguent aujourd'hui par le choix de filières dont sont en partie exclus les enfants des classes populaires (inégalités qualitatives) (Peugny, 2013).

L'hypothèse que nous faisons ici est que la démocratisation ségrégative s'opère non seulement in fine via la filière ou la spécialité de formation du diplôme final, mais aussi par la nature du parcours lui-même, principalement selon les types de formations qui ont jalonné ce parcours. En outre, les deux éléments sont liés, le parcours étant déterminant de la filière, voire de la spécialité du diplôme final.

La ségrégation associée à chaque filière est néanmoins souvent saisie sur le marché du travail par la valorisation du diplôme ou niveau final atteint. C'est une approche a priori assez logique, particulièrement pour évaluer les diplômes professionnels, puisque ces derniers sont censés permettre un accès direct au marché du travail, et non une poursuite d'études, réservée aux formations générales. La réalité est tout autre, avec trois logiques parfois contradictoires qui déterminent les parcours individuels. La première est celle de l'expansion scolaire qui est censée accroître la compétitivité des nations. Ainsi connait-on en France, successivement trois objectifs : $80 \%$ au bac (fixé en 1985, presque atteint en 2015 avec 77,5\%), puis 50 \% de diplômés du supérieur (HCEEE, 2006, actuellement $44 \%$ ) et très récemment $60 \%$ (Béjean et Montubert, 2015). Cet objectif n'est pas nouveau, même si les justifications ont évolué. Par exemple, les BTS (brevets de techniciens supérieurs) ou DUT (diplômes universitaires technologiques) ont été créés davantage pour répondre à des besoins immédiats, pendant une période qui s'accompagnait d'évolutions technologiques impactant considérablement l'évolution de la structure des qualifications : les Trente Glorieuses. Il s'agit maintenant d'anticiper des besoins futurs, ou de les créer par une dynamique d'innovation censée reposer sur l'accumulation de diplômés². Une spécificité de la politique française d'expansion scolaire a été de favoriser la professionnalisation des diplômes par la création de diplômes professionnels ou l'augmentation des effectifs accueillis dans les filières déjà existantes (Lemistre, 2015). Ainsi, l'enseignement supérieur en France a connu deux principaux mouvements de professionnalisation. Le premier débute dans les années 1960-1970 avec la création des BTS, DUT et DESS. Le début de la seconde ère de professionnalisation coïncide avec la création des licences professionnelles et le passage au LMD au début des années 2000. Après une multiplication des effectifs par neuf en BTS et plus de deux en DUT des années 1980 aux années 2000, ces effectifs ont ensuite stagné, avec 242000 diplômés en 2010 en BTS et 117000 diplômés en 2010 en DUT. L'expansion scolaire repose désormais sur les niveaux L (Licence) et M (Master). $\mathrm{Au}$ niveau L, la croissance des diplômes délivrés est portée par la licence professionnelle avec 44000 diplômes délivrés en 2010. Si au niveau M, la croissance du nombre de diplômes professionnels délivrés avant 2000 a été tirée par les bac+5 professionnels universitaires (les DESS alors), c'est sans commune mesure avec les évolutions à partir des années suivantes qui conduiront de 2000 à 2010 à presque 
doubler le nombre de diplômés de Master professionnel, pour atteindre 106000 diplômes de Master délivrés en 2010 (MENESR, 2016).

6 Pour permettre cette hausse des niveaux d'éducation, les parcours se modifient, ainsi que le rôle des diplômes au sein de ces parcours, particulièrement des diplômes professionnels. Destinés tout d'abord à permettre un accès direct au marché du travail, ces derniers deviennent propédeutiques, au moins jusqu'à bac+3. Par exemple, les baccalauréats technologiques sont d'abord destinés à une insertion immédiate. Ensuite, les brevets de techniciens supérieurs s'inscrivent dans la continuité de ces baccalauréats technologiques, de même que les diplômes universitaires technologiques. Si les premiers vont assez bien assurer cette continuité, notamment car ils sont dispensés au sein des mêmes établissements (lycées technologiques), ce ne sera rapidement pas le cas des seconds. La raison est simple: les meilleures filières (ou supposées) du supérieur attirent les bons lycéens, non seulement issus des filières technologiques, mais aussi des filières générales. Même si pour ces dernières il y a d'autres filières d'excellence (les classes préparatoires notamment), le DUT demeure un débouché privilégié par rapport à nombre de formations générales universitaires de premier cycle non sélectif. Ainsi les bacheliers généraux vont-ils devenir majoritaires parmi les sortants de DUT, dans le cadre de l'expansion scolaire. Corollaire de cette dernière, la sélectivité va conduire les étudiants sélectionnés en DUT à souhaiter poursuivre leurs études, puisqu'ils sont relativement performants. Un diplôme qui n'était pas destiné à la poursuite d'études le devient alors contre la volonté de l'institution, dans un premier temps du moins ${ }^{3}$. Dans ce contexte, plus de $60 \%$ des détenteurs de DUT poursuivent leurs études, avant même la création des licences professionnelles en 1999 , et plus de $80 \%$ pour les sortants de 2010 dont $50 \%$ jusqu'au niveau bac $+5^{4}$. Les Licences professionnelles semblent s'inscrire dans la même logique avec déjà $20 \%$ de poursuites d'études dans certaines universités (Gautié, 2016).

7 La dernière logique qui construit les parcours individuels, corollaire de la précédente (la sélectivité), est l'inégalité des chances, contre laquelle le continuum de bac-3 à bac+3 est censé lutté. Or, la sélectivité qui permet d'accéder à certaines filières interroge le réalisme de ce type de politiques publiques, pour lesquelles les filières professionnelles devaient constituer une seconde chance. Par exemple, en 2007 le document d'orientation du plan pluriannuel de réussite en licence (PRL-2007) précisait déjà que le PRL doit « ouvrir les filières professionnelles (STS, IUT, Licence Pro) pour favoriser la réussite de tous les étudiants». Pour la licence professionnelle, cela signifie que les étudiants en difficulté en licence générale devraient pouvoir bifurquer vers cette formation pour acquérir un diplôme de sortie. Nous verrons que les parcours qui permettent d'accéder à cette certification ne vont pas dans ce sens. De plus, les trajectoires demeurent déterminées par l'origine sociale qui influence la réussite à chaque niveau d'études et la décision de poursuite d'études.

Dans ce domaine, la France n'est pas parmi les pays les plus vertueux en matière de reproduction sociale 5 . Par exemple, un jeune sur trois dans la tranche d'âge des 18-23 ans était fille ou fils d'ouvrier en 2013. Or, l'année universitaire 2013/2014, les enfants d'ouvriers représentent environ un étudiant sur dix parmi l'ensemble des étudiants en Licence $(12,6 \%)$, et bien moins encore pour les Masters $(7,7 \%)^{6}$. Si le déterminisme social est fort, certains jeunes des classes populaires accèdent à l'université aux parcours Licence et au-delà. Ce sont les performances scolaires qui permettent à certains jeunes de bénéficier du processus sélectif précédemment décrit. Performances 
scolaires et reproduction sociale participent à l'élitisme républicain à la française (Baudelot et Establet, 2009). Il faut néanmoins garder à l'esprit que les performances scolaires demeurent en partie liée à l'origine sociale (Bourdieu et Passeron, 1964), d'une part. D'autre part, la sélectivité des filières est le ferment de la démocratisation ségrégative qui trouve ses sources dans le capital culturel des classes supérieures, mieux adaptées au fonctionnement du système éducatif et mieux informées sur ce dernier. L'origine sociale conduit alors à former des aspirations à la poursuite d'études différenciées. Le concept d'aspiration est actuellement fortement réapproprié par la sociologie, y compris dans le cadre de la sociologie de Pierre Bourdieu qui en rejetait une acception inspirée de Raymond Boudon (Allouch, 2016). Les aspirations ne sont pas alors envisagées comme des décisions individuelles, mais comme des faits sociaux qu'il faut rapporter à leur condition sociale de production (Orange, 2013, p. 6). Dans cette perspective, le processus de socialisation conduit les jeunes à former des vœux dans le système éducatif largement conformes aux représentations de leur milieu social, contribuant à générer "l'illusion biographique » (Bourdieu, 1986).

9 Tous les éléments précédents conduisent à un ensemble de parcours où les diplômes qui jalonnent le parcours et le niveau de sortie du système éducatif sont le reflet de la démocratisation ségrégative, un peu corrigée par la méritocratie qui s'exprime au fil de la sélectivité des parcours. Pour un même niveau final atteint dans une filière donnée, plusieurs parcours vont coexister, même si souvent un seul domine. Dès lors que le renforcement d'un continuum de bac-3 à bac+3 s'appuyant sur des parcours différenciés est affiché comme objectif, on peut alors s'interroger, d'une part sur le caractère discriminant d'une logique de parcours distincts, et d'autre part sur l'impact d'un parcours différent pour aboutir au même type de certification finale. Dans ce domaine, nous distinguerons deux types de parcours pour un même type de diplôme final : le parcours professionnalisé et le parcours général. Un jeune qui aura effectué un parcours avec un ou des diplômes professionnels successifs, associés in fine à une filière professionnelle ou générale, aura connu un parcours professionnalisé (voir Encadré 1).

10 Il s'agit d'élargir le cadre de la démocratisation ségrégative, où seules la filière et la spécialité du diplôme final reflètent un rang dans la hiérarchie des niveaux de diplômes. Ici, le parcours est susceptible de différencier la population selon d'origine sociale et en vue de l'insertion, pour un même type de diplôme final.

11 À cette fin, les parcours d'études ont été reconstitués à partir de l'enquête de $2013 \mathrm{du}$ CEREQ. Les parcours jusqu'à bac +5 et plus sont pris en compte, la poursuite d'études audelà de bac+3 participant de l'aboutissement du parcours bac-3 à bac+ 3. De plus, analyser les parcours vise évidemment surtout à caractériser les ségrégations pour ceux qui atteignent le supérieur. Cet article est donc consacré aux sortants du supérieur.

Dans cette perspective, l'objectif de cet article est double. Tout d'abord, il s'agit d'établir un état des lieux des parcours et leur lien à l'origine sociale. Ainsi, le premier développement est consacré à l'identification des trajectoires plus ou moins professionnalisées et à leur sélectivité. Le second développement met en évidence les liens entre trajectoires et capitaux économiques et culturels, saisis respectivement par la profession et le niveau d'études des parents. Enfin, sera interrogée la prolongation sur le marché du travail de la ségrégation dans le système éducatif associée à la professionnalisation des parcours. Les parcours de la génération diplômée en 2010 
seront mis en regard du nombre de mois au chômage au cours des trois premières années de vie active et de la qualification à la fin de ces trois années en 2013.

\section{Trajectoires individuelles et origine sociale}

\section{Encadré 1. Données et méthode}

\section{Échantillon retenu et trajectoires présentées}

Les investigations empiriques s'appuient sur l'exploitation de l'enquête du CEREQ de 2013 qui permet à la fois de saisir les parcours d'études par filières, et la situation sur le marché du travail, trois ans après la sortie du système éducatif de la génération des sortants de 2010. Les enquêtes Génération du CEREQ sont des enquêtes rétrospectives où le jeune est invité à reconstituer son parcours professionnel et d'études trois ans après sa sortie du système éducatif.

L'échantillon retenu ici ne concerne que les sortants qui ont atteint un niveau de fin d'études dans le supérieur, diplômé ou non, en 2010. Par exemple, un nondiplômé de Brevet de technicien supérieur est sortant de deuxième année de BTS, mais n'a pas obtenu le diplôme. Pour les Licences générales, les sortants non diplômés sont issus de la troisième année. L'échantillon comprend 17490 sortants du supérieur représentatifs de 317166 individus (des non-diplômés pour les certifications à bac+2 aux diplômés de Doctorat en 2010). Trente-neuf trajectoires ont été construites à partir des diplômes successifs déclarés par les individus à partir de regroupements en fonction des effectifs (non pondérés), ceci en fixant le seuil minimal à 100 individus à de rares exceptions près. La construction des trajectoires vise à identifier les diplômes professionnels intermédiaires constitutifs d'un parcours dit professionnalisé ici, par opposition à un parcours général qui n'est émaillé que de diplômes généraux. Par exemple, un Master parcours général sous-tend a minima : bac général puis Licence générale avant le Master. Il importe de noter que le qualificatif « professionnalisé » s'applique à la trajectoire, que le diplôme final soit un diplôme professionnel ou non. Sur trente-neuf, seules vingttrois trajectoires de sortants du supérieur sont reproduites (Tableau 1) pour simplifier la lecture et aussi afin de se focaliser uniquement sur les niveaux d'études les plus courants qui se distinguent en regard du parcours. Ainsi ne sont pas reproduites les trajectoires des bac +4 diplômés et non diplômés (DIP et ND désormais), les trajectoires « autres bac+2 », 3 et 5 (DIP et ND), et les non diplômés de Licences professionnelles et d'écoles de commerce (effectifs trop faibles pour l'analyse). Ne sont également pas reproduites les trajectoires associées à des professions réglementées en santé, soit les bac $+2 / 3$ en santé social (DIP et ND), ni les trajectoires uniformes des sortants de Doctorat.

L'origine sociale : capital économique et culturel

Les termes de « capital économique » et « capital culturel » sont mobilisés respectivement pour les professions et niveaux d'études du père et de la mère. S'ils renvoient aux catégories de la sociologie de Bourdieu, ils en sont évidemment une version très simplifiée. Le capital économique est bas si le père et la mère occupent au mieux des emplois d'ouvriers ou employés (dont : « père ou mère ne sais pas » 
et " père ou mère ouvrier ou employé »). Il est élevé si le père ou la mère ont un emploi de statut cadre. Le capital culturel est bas si le père et la mère ont un niveau d'études inférieur au bac (dont : « père ou mère ne sais pas » et " père ou mère < bac »). Il est élevé si le père ou la mère ont un niveau d'études supérieur au bac.

\section{Les indicateurs d'insertion}

Pour chaque trajectoire, deux indicateurs d'insertion ont été retenus : le nombre de mois de chômage de 2010 à 2013 pour l'ensemble de la population et le déclassement en 2013 pour les seuls individus en emploi à la date d'enquête. La norme de déclassement s'applique au diplôme obtenu, c'est pourquoi les trajectoires sont présentées par niveau de diplôme. La mesure du déclassement repose sur une norme de correspondance entre niveau de diplômes et de qualification, elle est la suivante pour les niveaux de diplômes reproduits dans le tableau 1 : les détenteurs d'un diplôme de niveau bac +5 sont déclassés s'ils occupent un emploi de statut non cadre. Les diplômés de niveau bac+2 et 3 sont déclassés s'ils sont ouvriers ou employés. Les diplômés des niveaux bac sont déclassés s'ils sont ouvriers non qualifiés ou employés non qualifiés. Il est important de noter que le plus haut diplôme d'un jeune qui n'a pas obtenu son BTS ou DUT est évidemment le bac, la norme qui s'applique à lui est donc la précédente, celle des bacheliers.

Les résultats qui figurent dans le tableau 1 sont descriptifs. Une analyse « toutes choses égales par ailleurs » a également été menée, mais elle apporte peu d'informations supplémentaires pour les deux variables retenues ici (Lemistre, 2017).

Tableau 1. Trajectoires, origine sociale et insertion

\begin{tabular}{|c|c|c|c|c|c|c|c|}
\hline \multirow{3}{*}{$\begin{array}{l}\text { Trajectoires de formation } \\
\text { initiale pour les jeunes } \\
\text { ayant atteint au moins le } \\
\text { niveau bac }+2 \\
\text { (diplômé ou non } \\
\text { diplômé) } \\
\text { (bac pour comparaison) }\end{array}$} & \multirow{3}{*}{$\begin{array}{l}\text { Effectif } \\
\text { (1) }\end{array}$} & \multicolumn{2}{|c|}{$\begin{array}{l}\text { Capital } \\
\text { économique }\end{array}$} & \multicolumn{2}{|c|}{$\begin{array}{l}\text { Capital } \\
\text { culturel }\end{array}$} & \multirow{3}{*}{$\begin{array}{l}\text { Nombre } \\
\text { de mois } \\
\text { au } \\
\text { chômage } \\
\text { de } 2010 \text { à } \\
2013 \\
\text { (moyenne) }\end{array}$} & \multirow{3}{*}{$\begin{array}{l}\text { Déclassement en } \\
2013 \quad \text { (population } \\
\text { en emploi) (2) }\end{array}$} \\
\hline & & \multicolumn{2}{|c|}{$\begin{array}{l}\text { Père et/ou* } \\
\text { mère }\end{array}$} & \multicolumn{2}{|c|}{$\begin{array}{l}\text { père et/ou* } \\
\text { mère }\end{array}$} & & \\
\hline & & $\begin{array}{l}\text { Ouv. } \\
\text { Emp. }\end{array}$ & Cadre* & $<\mathrm{Bac}$ & $>\mathrm{Bac}^{*}$ & & \\
\hline Plus haut diplôme BAC & & & & & & & \\
\hline $\begin{array}{l}\text { (3) ND BTS ou DUT Bac } \\
\text { Pro }\end{array}$ & $2,9 \%$ & $63 \%$ & $16 \%$ & $63 \%$ & $12 \%$ & 7,1 mois & $30 \%$ \\
\hline ND BTS ou DUT Bac Tech & $3,4 \%$ & $53 \%$ & $22 \%$ & $52 \%$ & $24 \%$ & 7,5 mois & $24 \%$ \\
\hline ND BTS ou DUT Bac Gen & $2,6 \%$ & $48 \%$ & $31 \%$ & $44 \%$ & $34 \%$ & 6,3 mois & $21 \%$ \\
\hline $\begin{array}{l}\text { Plus haut diplôme } \\
\mathrm{BAC}+2\end{array}$ & & & & & & & \\
\hline
\end{tabular}




\begin{tabular}{|c|c|c|c|c|c|c|c|}
\hline BTS ou DUT Bac Pro & $3,7 \%$ & $55 \%$ & $18 \%$ & $60 \%$ & $17 \%$ & 5,1 mois & $43 \%$ \\
\hline (4) BTS ou DUT Bac Tech. & $10,1 \%$ & $48 \%$ & $25 \%$ & $55 \%$ & $23 \%$ & 6,3 mois & $45 \%$ \\
\hline BTS ou DUT Bac Gen & $6,4 \%$ & $39 \%$ & $36 \%$ & $43 \%$ & $36 \%$ & 4,8 mois & $38 \%$ \\
\hline ND LG BTS ou DUT & $0,7 \%$ & $48 \%$ & $24 \%$ & $51 \%$ & $30 \%$ & 6,9 mois & $42 \%$ \\
\hline ND Licence Générale Bac & $1,7 \%$ & $33 \%$ & $44 \%$ & $45 \%$ & $40 \%$ & 5,4 mois & $27 \%$ \\
\hline \multicolumn{8}{|l|}{$\begin{array}{l}\text { Plus haut diplôme } \\
\mathrm{BAC}+3\end{array}$} \\
\hline $\begin{array}{l}\text { LP BTS ou DUT Bac Pro } \\
\text { ou Tech }\end{array}$ & $3,2 \%$ & $41 \%$ & $28 \%$ & $45 \%$ & $30 \%$ & 4,6 mois & $22 \%$ \\
\hline LP BTS ou DUT Bac Gen & $2,7 \%$ & $35 \%$ & $41 \%$ & $36 \%$ & $42 \%$ & 4,2 mois & $20 \%$ \\
\hline LP autres bac +2 & $0,7 \%$ & $37 \%$ & $34 \%$ & $47 \%$ & $40 \%$ & 3,8 mois & $21 \%$ \\
\hline LG Bac Pro ou Tech & $0,6 \%$ & $37 \%$ & $40 \%$ & $40 \%$ & $42 \%$ & 5,6 mois & $33 \%$ \\
\hline LG Bac Gen & $4,1 \%$ & $37 \%$ & $43 \%$ & $36 \%$ & $47 \%$ & 4,3 mois & $33 \%$ \\
\hline LG BTS ou DUT & $1,4 \%$ & $36 \%$ & $36 \%$ & $45 \%$ & $35 \%$ & 4,4 mois & $33 \%$ \\
\hline ND Master 2 & $1,1 \%$ & $38 \%$ & $45 \%$ & $46 \%$ & $38 \%$ & 4,4 mois & $17 \%$ \\
\hline \multicolumn{8}{|l|}{$\begin{array}{l}\text { Plus haut diplôme } \\
\mathrm{BAC}+5\end{array}$} \\
\hline Master DUT BTS & $2,8 \%$ & $34 \%$ & $42 \%$ & $37 \%$ & $46 \%$ & 4 mois & $40 \%$ \\
\hline Master Licence Pro & $1,2 \%$ & $35 \%$ & $41 \%$ & $35 \%$ & $48 \%$ & 5,2 mois & $40 \%$ \\
\hline (5) Master parcours Gen & $17 \%$ & $25 \%$ & $55 \%$ & $27 \%$ & $58 \%$ & 4,7 mois & $36 \%$ \\
\hline $\begin{array}{l}\text { (5) École Com Parcours } \\
\text { Pro }\end{array}$ & $1,1 \%$ & ns & $52 \%$ & $32 \%$ & $53 \%$ & 4,3 mois & $40 \%$ \\
\hline $\begin{array}{l}\text { (5) École Com Parcours } \\
\text { Gen }\end{array}$ & $2,0 \%$ & ns & $65 \%$ & $18 \%$ & $72 \%$ & 3,3 mois & $28 \%$ \\
\hline $\begin{array}{l}\text { (5) Ingénieur parcours } \\
\text { Pro }\end{array}$ & $2,0 \%$ & $27 \%$ & $44 \%$ & $40 \%$ & $45 \%$ & 2,3 mois & $11 \%$ \\
\hline
\end{tabular}




\begin{tabular}{|l|l|l|l|l|l|l|l|}
\hline $\begin{array}{l}\text { (5) Ingénieur Parcours } \\
\text { Général }\end{array}$ & $3,7 \%$ & $20 \%$ & $61 \%$ & $18 \%$ & $67 \%$ & 2,4 mois & $12 \%$ \\
\hline
\end{tabular}

(1) Les pourcentages concernent les trajectoires du supérieur telles que définies dans l'encadré. Celles reproduites ici représentent $75,1 \%$ des sortants retenus, les $24,9 \%$ restants concernent les trajectoires non reproduites (voir encadré).

(2) Pour le déclassement, voir la norme de correspondance en encadré.

(3) ND BTS ou DUT Bac Pro : sortants en 2010 de deuxième année de DUT ou BTS non diplômés,

ayant obtenu en amont un baccalauréat professionnel.

(4) Lire :

- les détenteurs d'un BTS ou d'un DUT ayant obtenu en amont un baccalauréat technologique représentent $10,1 \%$ des jeunes ayant atteint au moins le niveau bac+2 de la génération 2010 - pour cette trajectoire : 48 \% ont un père et une mère ouvrier ou employé (voir détail des catégories en encadré), $25 \%$ ont au moins un des deux parents qui a une profession de statut-cadre, $55 \%$ ont un père et une mère qui ont un diplôme inférieur au bac, 23 \% ont un père ou une mère qui ont un diplôme supérieur au bac.

- pour cette trajectoire la durée moyenne de chômage à la sortie du système éducatif entre 2010 et 2013 est de 6,3 mois. 45 \% des jeunes en emploi en 2013 de cette trajectoire sont déclassés. Pour un diplôme de niveau bac +2 , cela signifie que $45 \%$ de ces jeunes sont ouvriers ou employés (voir norme en encadré).

(5) Parcours pro, soit professionnalisé : au moins un diplôme professionnel dans le parcours. II peut s'agir d'un DUT ou d'un BTS ou d'un baccalauréat technologique ou professionnel, ou de plusieurs de ces diplômes.

Parcours Gen, soit général : aucun diplôme professionnel dans le parcours antérieur.

Source : enquête 2013 du CEREQ (génération 2010) calculs de l'auteur

\section{1) Des trajectoires plus ou moins professionnalisées et toujours sélectives}

13 Les trajectoires ont été regroupées pour permettre une analyse en regard des capitaux économiques et culturels et des indicateurs d'insertion. Cette première section examine ces trajectoires en donnant quelques éléments de détail pour chacune d'entre elles afin d'illustrer la sélectivité des parcours.

14 Comme cela a été évoqué, la sélectivité des parcours conduit de nombreux diplômés de DUT et aussi de BTS à poursuivre leurs études. Les sortants du système éducatif en 2010 immédiatement après le BTS et de DUT ne représentent donc pas la majorité des formés de ces filières dont on retrouvera les diplômés dans les trajectoires de niveau supérieur. Ces sortants de niveau BTS-DUT représentent $29,1 \%$ des étudiants ayant été au terme d'un cycle du supérieur, dont $8,9 \%$ de non-diplômés. Pour les diplômés, près d'un détenteur sur deux d'un BTS ou d'un DUT sortant à ce niveau est issu d'un bac technologique. Viennent ensuite un tiers de bacs généraux (32\%), puis les bacs pros qui représentent $18 \%$ de ces diplômés. Si l'on détaille la trajectoire «BTS ou DUT bac pro » (Tableau 1), 98 \% de ces sortants de BTS ou DUT diplômés et détenteurs d'un bac pro sont issus de BTS, $92 \%$ pour les bacs technologiques (trajectoire BTS ou DUT bac Tech) et $73 \%$ pour les bacs généraux (trajectoire BTS ou DUT bac Tech). La très grande majorité des sortants des BTS et DUT sont donc logiquement issus de BTS, les sortants de DUT poursuivant leurs études dans une plus forte proportion.

Pour la très grande majorité des diplômés de bac pro, bac+2 est le plus haut niveau atteint dans le supérieur, au-delà ils sont diplômés uniquement de Licence professionnelle, où ils représentent seulement $6 \%$ des sortants ( $6 \%$ de toutes les trajectoires plus haut diplôme bac+3 LP, Tableau 1), tous avec un BTS ou un DUT au préalable (trajectoire «LP BTS ou DUT Bac Pro ou Tech», Tableau 1). Ce sont les bacs 
généraux qui dominent en Licence professionnelle, traduisant la sélectivité de cette filière professionnelle. Tout d'abord, car bacheliers généraux et technologiques détenteurs d'un BTS ou d'un DUT représentent un effectif comparable parmi les diplômés de licence professionnelles ${ }^{7}$. Ensuite, car les $80 \%$ des étudiants qui entrent en licence professionnelle sans BTS ou DUT sont détenteurs d'un bac général. Il s'agit évidemment essentiellement des étudiants issus d'une filière générale de l'université. Ce ne sont pas des étudiants en difficulté scolaire : $40 \%$ ont eu une mention au bac, soit exactement la même proportion que les bacs généraux qui ont accédé à la Licence professionnelle après un BTS ou un DUT. Il n'y a donc pas de seconde chance, quel que soit le parcours à bac +2 . En outre, ce parcours sans filière sélective au préalable ne représente que $10 \%$ des trajectoires vers la Licence professionnelle.

La sélectivité se renforce pour les niveaux supérieurs à bac+3 où les bacheliers professionnels sont marginaux (moins de 0,5\%, essentiellement parmi les nondiplômés). Quant aux bacheliers technologiques diplômés à bac +5 , ils ne représentent que $9 \%$ de l'ensemble des diplômés de ce niveau (ensemble des trajectoires plus haut diplôme bac+5) et sont quasiment tous issus de BTS ou DUT. Parmi les détenteurs de BTS ou de DUT, ce sont donc les bacs généraux qui dominent nettement au sein des diplômés à bac+5, par exemple à hauteur de $93 \%$ pour les diplômés de Master (\% de bac généraux de la trajectoire " Master DUT-BTS »).

passage par un DUT ou un BTS est en effet surtout une bifurcation pour de jeunes bacheliers généraux souhaitant vraisemblablement contourner les premiers cycles universitaires généraux, non seulement à bac +5 , mais aussi à bac +3 où le passage par un DUT ou un BTS concerne plus de $20 \%$ des trajectoires des sortants diplômés de Licence générale ${ }^{8}$.

Certains vont même poursuivre cette stratégie de contournement de la filière générale jusqu'à la Licence professionnelle pour rejoindre le Master. Ainsi, les parcours professionnalisés avant l'obtention du Master (DUT ou BTS et/ou Licence pro, Tableau 1) représentent près de $20 \%$ des diplômés de Master (Tableau 1, parcours professionnalisé / tous parcours Masters soit : $4 \%$ / $21 \%$ ). Plus encore pour les écoles de commerce ou d'ingénieur, où les parcours professionnalisés concernent un tiers des diplômés. Pour les écoles de commerces plus de 90 \% sont des parcours post DUT-BTS ou Licence professionnelle. Pour les écoles d'ingénieurs $75 \%$ viennent de DUT-BTS, $10 \%$ de Licence générale et les autres de procédures d'admission post bac en école d'ingénieur. Plus d'un tiers des jeunes en école d'ingénieur ayant un parcours professionnalisé détiennent un baccalauréat technologique. Il semble donc que l'on trouve ici une possible préfiguration d'une filière professionnelle du bac à bac+5. Reste à examiner le caractère ségrégatif de ces trajectoires professionnalisées et des autres, et leurs répercussions à l'insertion.

\section{2) Démocratisation ségrégative et parcours professionnalisés}

19 L'accès aux différentes filières du supérieur s'avère donc très sélectif en fonction du type de bac et du parcours dans le premier cycle du supérieur. Cette sélectivité pourrait néanmoins sembler équitable, si elle était uniquement liée aux performances des jeunes dans le système éducatif. L'accès aux filières ne serait donc ségrégatif qu'en vertu d'un principe méritocratique. L'hypothèse de démocratisation ségrégative induit néanmoins d'une part un lien entre performances scolaires et origine sociale et, d'autre part, des 
choix de parcours clairement différenciés selon cette même origine sociale, quelles que soient les performances scolaires. Le lien entre les trajectoires et les capitaux économiques et culturels des parents conforte ces hypothèses (voir Encadré 1 pour la définition de ces capitaux).

Plus le parcours est professionnalisé (pro>techno>gén) et plus les capitaux économiques et culturels sont modestes, avec souvent un effet renforcé du capital culturel par rapport au capital économique. Par exemple pour les diplômés de BTS ou de DUT, les pourcentages de jeunes dont le père et la mère sont ouvriers ou employés sont pour les bacs généraux de $39 \%$, pour les bacs technos de $48 \%$ et pour les bacs pros de $55 \%$ (trajectoires BTS ou DUT bac gen, bac tech, bac pro ; Tableau 1, colonne « Ouv. Emp »). Quant au capital culturel, le pourcentage de jeunes sortants diplômés de BTS ou DUT dont l'un des deux parents a un niveau d'études du supérieur est pour les détenteurs de bacs généraux de $36 \%$, pour les bacs technos $23 \%$, et $17 \%$ pour les bacs pros (trajectoires BTS ou DUT bac gen, bac tech, bac pro ; Tableau 1, colonne « >bac »).

L'obtention du BTS ou du DUT est également toujours en faveur des plus dotés en capitaux, quel que soit le type de bac qui mène au niveau BTS ou DUT. Par exemple, $63 \%$ des non-diplômés de BTS-DUT issus d'un bac pro ont un père et une mère qui ont au plus une qualification d'ouvrier ou d'employé, contre 55 \% des diplômés de BTS ou DUT issus de ce même type de bac (Tableau 1).

22 Au niveau immédiatement supérieur, $45 \%$ des diplômés de Licence professionnelle ayant eu au préalable un BTS ou un DUT et un bac technologique ou professionnel, ont un père et une mère avec un diplôme inférieur au bac (LP BTS DUT bac pro ou tech ; Tableau 1, colonne «<bac»). Un pourcentage nettement inférieur à celui des détenteurs de l'un des ces bacs qui n'ont pas poursuivi au-delà du BTS ou du DUT. Par exemple, les jeunes diplômés de BTS et DUT ayant un bac technologique ont pour $55 \%$ d'entre eux un père et une mère avec un diplôme inférieur au bac (60 \% pour les bacs pro). Ce constat est aussi le résultat d'une sélectivité sociale des filières en amont, la proportion de sortants de DUT parmi les licences professionnelles étant très supérieure à la proportion de sortants de DUT au niveau bac+2 par rapport au BTS. Or, les détenteurs de DUT sont plus en dotés capitaux économiques et culturels que les BTS (Orange, 2014).

23 Pour les bac +5 , être issu d'un parcours professionnalisé (au moins un diplôme professionnel dans la trajectoire) s'accompagne de moindres capitaux économiques et culturels que le parcours général, particulièrement pour les écoles de commerce ou d'ingénieur. Par exemple, les diplômés de Master ayant eu un parcours sans diplômes professionnels (Master parcours gen) ont pour $58 \%$ d'entre eux un père ou une mère dont le niveau d'études est supérieur au bac (Tableau 1, « colonne >bac »), contre $46 \%$ pour les jeunes qui ont dans leur parcours obtenu un BTS ou un DUT (trajectoire Master DUT-BTS). Cet écart de 12 points ( $58 \%$ - $46 \%$ ) est porté, respectivement pour les écoles de commerce et d'ingénieur, à 19 points $(72 \%-53 \%)$ et 22 points $(67 \%-45 \%)$, si l'on compare les parcours généraux aux parcours professionnalisés.

\section{3) Trajectoires et insertions : les trajectoires les plus rentables pour les plus dotés en capital social?}

Cette ségrégation à la fois méritocratique et sociale se poursuit en partie sur le marché du travail. Une explication est que les capitaux culturels et surtout économiques sont 
déterminants de la qualité de l'insertion. Par exemple, à bac+2, parmi les détenteurs de BTS ou de DUT, les bacheliers généraux, nettement plus dotés en capitaux économiques et culturels que les bacheliers technologiques (cf. supra), ont une insertion plus favorable que ces derniers ( $7 \%$ de déclassés et 1,5 mois de chômage en moins). On retrouve la faveur à l'insertion des plus dotés socialement en examinant les deux trajectoires de non-diplômés de Licence générale. Tout d'abord, continuer en Licence générale avec un BTS ou un DUT n'apporte pas de plus-value par rapport à une sortie immédiatement après le BTS ou le DUT (trajectoire ND LG BTS ou DUT / trajectoires BTS ou DUT). Or, ces jeunes non diplômés de licence générale ont un capital économique et culturel qui n'est pas favorable par rapport aux diplômés de BTS et DUT, et même un capital économique nettement défavorable par rapport aux diplômés de BTS DUT issus de bac généraux (cf. supra). Ensuite, et a contrario, sortir de troisième année de Licence générale non diplômé sans BTS ou DUT (ND Licence générale Bac) conduit à une insertion plus favorable que tous les autres parcours à bac+2. Là encore, l'origine sociale joue un rôle déterminant, car à ce niveau d'études c'est la trajectoire où il y a le plus d'enfants de cadres, soit $44 \%$. Or, ce pourcentage est très supérieur aux 20 \% d'enfants de cadre pour la trajectoire des non-diplômés de Licence générale issus de BTS et DUT.

À bac+3, la Licence professionnelle apporte une plus-value moyenne manifeste par rapport aux diplômés de BTS et DUT, particulièrement pour le déclassement, du simple au double pour les détenteurs de bacs pros ou technos. Par exemple, $38 \%$ des diplômés de BTS DUT ayant un bac général n'accèdent pas à la qualification de technicien ou profession intermédiaire, contre $20 \%$ de ceux qui ont complété ce parcours avec une Licence professionnelle. Or, à type de bac comparable, les capitaux économiques et culturels sont cette fois nettement favorables aux diplômés de Licences professionnelles par rapport aux sortants de BTS ou DUT. En revanche, et c'est une exception notable, les diplômés de Licence professionnelle ayant un BTS ou un DUT ont une insertion proche, quel que soit le type de bac, alors que les capitaux économiques et culturels sont nettement favorables aux bacheliers généraux. La sélectivité des BTS ou DUT semble donc permettre de pallier les différences en termes d'origine sociale. Il faut néanmoins garder à l'esprit que la proportion de bacheliers généraux est nettement plus forte en Licence professionnelle qu'en DUT ou BTS, traduisant une sélection plus forte des bacheliers technologiques ou professionnels, ou plutôt un choix de poursuite d'études nettement moins prononcées pour ces bacheliers (cf. supra). En clair, la démocratisation ségrégative est avérée, mais n'a pas cette fois d'incidence à l'insertion pour les jeunes issus des filières où ils sont les moins dotés en capitaux économiques et culturels.

Le même constat vaut pour les sortants de Master : les parcours professionnels y sont ici plus minoritaires encore que pour les diplômés de Licence professionnelle, mais pour les sélectionnés en Master issus de parcours professionnalisés (LP ou DUT ou BTS) l'insertion n'est que très légèrement défavorable en matière de déclassement, alors que les écarts en termes d'origine sociale sont à la défaveur des parcours professionnels. Si le constat est le même pour les écoles d'ingénieurs, avec des écarts en termes de capitaux économiques et culturels nettement plus accentués, ce n'est pas le cas pour les écoles de commerce. Un parcours professionnalisé (un diplôme professionnel au moins dans le parcours) avant l'obtention du diplôme d'écoles de commerce conduit $40 \%$ des jeunes issus de cette trajectoire à ne pas accéder à l'emploi-cadre contre $28 \%$ pour le 
parcours général. Là encore la spécificité est l'origine sociale. Les diplômés d'écoles de commerces au parcours général appartiennent en effet à la trajectoire qui à la plus forte proportion de parents-cadres (65\%) et diplômés du supérieur (72 \%).

\section{Conclusion}

Dès les années 1970, Pierre Bourdieu (1979) soulignait le caractère socialement ségrégatif des filières et spécialités de formation du secondaire au détriment des jeunes d'origine sociale modeste. Trois décennies plus tard, Pierre Merle (2012) met en évidence la démocratisation ségrégative. L'expansion scolaire permet, certes, d'amener des enfants de milieux sociaux modestes à des niveaux de formation de plus en plus élevés, mais pas dans les mêmes proportions que les plus dotés socialement, ni dans les mêmes filières et spécialités. Nous avons saisi cette ségrégation d'une manière un peu différente qu'habituellement, soit par la seule spécialité ou filière du diplôme final. L'accent a été mis sur le caractère professionnalisé du parcours antérieur, par opposition à un parcours général où ne figure aucun diplôme professionnel. Le lien entre parcours professionnalisé et origine sociale apparaît très clairement, que le diplôme final soit professionnel ou non. Plus le parcours est professionnalisé et plus l'origine sociale est modeste. On retrouve notamment les ségrégations en défaveur des bacheliers technologiques et professionnels à travers les différents "tris » effectués dans le supérieur. Les jeunes aux parcours professionnalisés du secondaire sont en effet défavorisés, tant pour l'accès aux différents niveaux du supérieur que pour l'obtention des diplômes. Quant à la seconde chance qui devrait être offerte par les filières professionnelles du supérieur selon plusieurs textes institutionnels (le plan réussite en Licence notamment), la sélectivité de ces dernières ne la garantit absolument pas. Par exemple, la faible part de jeunes qui accède à la Licence professionnelle sans être passé par une filière sélective de type BTS ou DUT n'est ni issue de bacs technologiques ou professionnels ni constituée de jeunes aux faibles performances scolaires antérieures. Plus généralement, les jeunes qui accèdent actuellement aux filières professionnelles du supérieur ne sont en aucun cas les plus vulnérables, ce sont des jeunes d'origine sociale favorisée ou qui ont été performants dans un parcours du secondaire très souvent général.

Par ailleurs, plus l'accès à un diplôme du supérieur est sélectif, plus le diplôme devient propédeutique. En effet, comme en son temps pour les DUT, la proportion de jeunes en poursuite d'études après une Licence professionnelle augmente. Les diplômes professionnels constituent davantage une étape intermédiaire pour les plus dotés en capitaux économiques et culturels ou les plus performants dans le secondaire, qu'un recours possible pour les plus en difficulté. Une logique d'ensemble qui met en évidence un nouvel élément contribuant à «l'élitisme " républicain (Baudelot et Establet, 2009).

Toutefois, si la démocratisation ségrégative s'applique au système éducatif via les parcours professionnalisés, est-ce qu'elle se poursuit sur le marché du travail ? Pour la génération de sortants du système éducatif de 2010 observée en 2013, à bac+5 les parcours professionnalisés avant le diplôme final ne sont plus pénalisants à l'entrée sur le marché du travail pour les Masters et les écoles d'ingénieur, au prix néanmoins d'une forte sélection des jeunes issus de filières professionnelles, et aussi à la condition que 
les écarts en termes de capitaux économiques et culturels ne soient pas trop prononcés, comme c'est le cas pour les écoles de commerce.

Est-ce que la volonté politique peut contrecarrer les logiques précédentes, propres au système éducatif français? Pour certains, c'est possible à moyen terme, à condition offrir des secondes chances en créant ou facilitant les passerelles entre formations, en formation initiale et au-delà, soit en "créant des marches" (Lichtenberger et Korolitski, 2012). Au vu des investigations réalisées, on peut s'interroger sur les critères qui permettront de gravir ces marches. Dans la continuité du fonctionnement actuel d'une méritocratie socialement reproductive, le système de tri actuel ne risque-t-il pas de se renforcer vers une extension de l'élitisme républicain toujours au détriment des plus vulnérables dans le système éducatif et sur le marché du travail ?

Si les résultats de cet article légitiment cette question, ils ne permettent pas d'y répondre. En effet, en focalisant l'analyse sur le caractère professionnalisé des parcours et compte tenu des contraintes d'effectifs, il n'a pas été possible d'expliciter les bifurcations ou leur absence liées aux différentes spécialités constitutives des parcours. En outre, les études sur données nationales ne permettent pas de désagréger suffisamment les spécialités pour permettre une telle analyse. Dans ce registre, contrairement au secondaire, les études qualitatives ou quantitatives sont encore assez rares et donc nécessaires pour le supérieur.

\section{BIBLIOGRAPHIE}

Allouch A. (2016), « De la notion d'aspiration en sociologie », in Van Zanten et Rayou (dir.), Dictionnaire de l'éducation, Paris, PUF.

Baudelot C. et Establet R. (2009), L'élitisme républicain. L'école française à l'épreuve des comparaisons internationales, Paris, Seuil.

Béjean S et Montubert B. (2015), «Pour une société apprenante : proposition pour une stratégie nationale de l'enseignement supérieur ", rapport STRANES, ministère de l'éducation nationale, de l'enseignement supérieur et de la recherche, $250 \mathrm{p}$.

Bourdieu P. (1986), « L'illusion biographique », Actes de la recherche en sciences sociales, vol. 62-63, p. 69-72.

Bourdieu P. (1979), La distinction, Paris, Minuit.

Boudieu P. et Passeron J.-C. (1964), Les héritiers. Les étudiants et la culture, Paris, Minuit.

Baudelot C. et Leclerc F., (2005), « Les effets de l'éducation », rapport à l'intention du PIREF, Paris, La Documentation Française.

Gautié C. (2016), «Professionnalisation et poursuite d'études », in Lemistre P. et Mora V. (dir.), Professionnalisation des publics et des parcours à l'université, Marseille, CEREQ, 2016, p. 79-91. 
Haut comité éducation-économie-emploi, (2006), « Objectif 50 \% d'une génération diplômée de l'enseignement supérieur », rapport du Haut comité éducation-économie-emploi, Paris, La Documentation française.

MENESR (dir.) (2016), État de l'enseignement supérieur et de la recherche en France - 50 indicateurs, Paris, ministère de l'Éducation nationale, de l'Enseignement supérieur et de la Recherche. En ligne : https://publication.enseignementsup-recherche.gouv.fr/eesr/7/info.php.

Lemistre P. (2017), « À qui profite la professionnalisation des parcours ? ", in Calmand J. et al. (dir.), Rendement éducatif, parcours et inégalités dans l'insertion des jeunes, Marseille, CEREQ, p. 109-129.

Lemistre P. (2015), « La professionnalisation des formations initiales : une solution aux mutations du marché de l'emploi pour les jeunes ? ", Revue française de pédagogie, n 192, p. 61-72.

Lichtenberger Y. et Korolitski J.-P. (2012), « Quelle valeur accorder à la licence, pour quels étudiants? ", in Administration et éducation, revue de l'AFAE, nº 133, p. 105-110.

Merle P. (2012), La ségrégation scolaire, Paris, La Découverte.

Orange S. (2013), L'autre enseignement supérieur. Les BTS et la gestion des aspirations scolaires, Paris, PUF.

Peugny C. (2013), Le destin au berceau. Inégalités et reproduction sociale, Paris, Seuil.

\section{NOTES}

1. Par exemple, on peut considérer comme professionnels que des diplômes de filières classifiées générales, telle que les licences générales de STAPS (sciences et techniques des activités physiques et sportives) ou d'AES (Administrations économiques et sociales). De plus, la visée professionnelle de la Licence générale a été introduite par un arrêté de 2011, précisant que ce diplôme en plus de préparer à la poursuite d'études, doit désormais également permettre l'insertion professionnelle immédiate.

2. Pour une synthèse des arguments en ce sens, voir Baudelot et Leclerc (2005).

3. Les poursuites d'études vont être institutionnalisées notamment à travers le nouveau PPN (programme pédagogique national des DUT) qui propose trois parcours de formation : insertion immédiate, poursuite courte (licence), poursuite longue (bac +5).

4. Source enquête DUT 2012.

5. Rapport OCDE (Organisation de coopération et de développement économique) de 2016.

6. Source MENESR (RRS 2014).

7. Part des bacs technologiques dans la trajectoire «LP BTS ou DUT Bac Pro ou Tech» égale effectif de la trajectoire «LP BTS ou DUT Bac Gen ».

8. Trajectoire LG BTS ou DUT, $1,4 \%$ par rapport à l'ensemble de trajectoire en licence générale : $1,4 \% /(0,6 \%+4,1 \%+1,4 \%)$. 


\section{RÉSUMÉS}

Selon Pierre Merle, si les jeunes d'origine sociale modeste haussent leur niveau d'éducation, c'est vers les diplômes de filières et spécialités les moins à même de permettre un accès privilégié au marché du travail. C'est la " démocratisation ségrégative ", dont cet article élargit le cadre aux sortants de l'enseignement supérieur, ceci en examinant le caractère ségrégatif des parcours d'études selon qu'ils comprennent des diplômés professionnels ou pas. La démocratisation ségrégative liée au parcours d'études suppose que, selon qu'ils soient professionnels ou non, les diplômes qui jalonnent ce parcours différencient la population en termes d'origine sociale et ont une influence sur la réussite au diplôme final, la poursuite d'étude et l'insertion. De fait, les investigations menées à partir de l'enquête du CEREQ de 2013 mettent en évidence le caractère ségrégatif des parcours professionnalisés.

According to Pierre Merle, if young people of modest social origin raise their level of education, it is towards the sectors and specialties least likely to allow privileged access to the labor market. It is "segregative democratization", which this article broadens to include higher education graduates by examining the segregative nature of educational pathways, whether or not they include professional graduates. The segregated democratization linked to the study path implies that the professional nature or not of the degrees of the study path, differentiates the population in terms of social origin and influences the success of the final diploma, the continuation of studies and access to the labor market. Indeed, the surveys conducted from the 2013 CEREQ survey highlight the segregation of vocational professionalized study pathways.

\section{INDEX}

Mots-clés : enseignement supérieur, ségrégation scolaire, marché du travail, diplôme, parcours d'études

Keywords : higher education, school segregation, labor market, diploma, study path

\section{AUTEUR}

\section{PHILIPPE LEMISTRE}

Chercheur CEREQ (Centre d'études et de recherches sur les qualifications) au CERTOP-UMR 5044 (université Toulouse Jean-Jaurès et CNRS, Centre d'étude et de recherche travail organisation pouvoir) 